\title{
The effects of ATP and sodium chloride on the cytochrome $c$-cardiolipin interaction: The contrasting behavior of the horse heart and yeast proteins
}

\author{
Federica Sinibaldi a, Enrica Droghetti ${ }^{\text {b }}$, Fabio Polticelli ${ }^{\text {c,d }}$, Maria Cristina Piro ${ }^{\text {a }}$, Donato Di Pierro ${ }^{\text {, }}$ \\ Tommaso Ferri ${ }^{\mathrm{e}}$, Giulietta Smulevich ${ }^{\mathrm{b}}$, Roberto Santucci ${ }^{\mathrm{a}, *}$ \\ a Department of Experimental Medicine and Biochemical Sciences, University of Rome 'Tor Vergata', Via Montpellier 1, I-00133 Rome, Italy \\ b Department of Chemistry "Ugo Schiff”, University of Florence, Via della Lastruccia 3-13, I-50019 Sesto Fiorentino, Italy \\ c Department of Biology, University Roma Tre, Viale Marconi 446, I-00146 Rome, Italy \\ d Institute of Nuclear Physics, Roma Tre Section Via della Vasca Navale 84, I-00146 Rome, Italy \\ e Department of Chemistry, University of Rome 'Sapienza', P.le A. Moro 5, I-00185 Rome, Italy
}

\section{A R T I C L E I N F O}

\section{Article history:}

Received 10 February 2011

Received in revised form 27 July 2011

Accepted 27 July 2011

Available online 4 August 2011

\section{Keywords:}

Cytochrome $c$

Cardiolipin

Mitochondrial membrane

Resonance Raman

Circular dichroism

\begin{abstract}
A B S T R A C T
In cells a portion of cytochrome $c$ (cyt $c)(15-20 \%)$ is tightly bound to cardiolipin (CL), one of the phospholipids constituting the mitochondrial membrane. The CL-bound protein, which has nonnative tertiary structure, altered heme pocket, and disrupted Fe(III)-M80 axial bond, is thought to play a role in the apoptotic process. This has attracted considerable interest in order to clarify the mechanisms governing the cyt $c-\mathrm{CL}$ interaction. Herein we have investigated the binding reaction of CL with the c-type cytochromes from horse heart and yeast. Although the two proteins possess a similar tertiary architecture, yeast cyt $c$ displays lower stability and, contrary to the equine protein, it does not bind ATP and lacks pro-apoptotic activity. The study has been performed in the absence and in the presence of ATP and $\mathrm{NaCl}$, two compounds that influence the (horse cyt $c$ )-CL binding process and, thus, the pro-apoptotic activity of the protein. The two proteins behave differently: while CL interaction with horse cyt $c$ is strongly influenced by the two effectors, no effect is observed for yeast cyt $c$. It is noteworthy that $\mathrm{NaCl}$ induces dissociation of the (horse cyt $c$ )-CL complex but has no influence on that of yeast cyt $c$. The differences found for the two proteins highlight that specific structural factors, such as the different local structure conformation of the regions involved in the interactions with either CL or ATP, can significantly affect the behavior of cyt $c$ in its reaction with liposomes and the subsequent pro-apoptotic action of the protein.
\end{abstract}

(C) 2011 Elsevier Inc. All rights reserved.

\section{Introduction}

Cytochrome $c$ (cyt $c$ ) is a single-chain hemoprotein acting as electron carrier in mitochondria. It is composed of 104 amino acids ( $12.4 \mathrm{kDa})$ and the prosthetic group is heme. In the protein the heme is covalently bound to the polypeptide chain by two thioether bridges formed with residues $\mathrm{C} 14$ and $\mathrm{C} 17$, while $\mathrm{H} 18$ and M80 are the two axial ligands of the heme iron. In the cellular environment the protein functions between the inner and the outer membrane of the mitochondrion, mediating electron transfer between different proteins of the respiratory chain. In healthy cells a portion of cyt $c(15-20 \%)$ remains tightly bound to cardiolipin (CL), one of the phospholipids constituting the mitochondrial membrane [1,2]. CL-bound cyt $c$ shows tertiary structural rearrangements, which include alteration of the heme pocket region and detachment of M80 from the sixth coordination position of the

\footnotetext{
* Corresponding author at: Dipartimento di Medicina Sperimentale e Scienze Biochimiche, Università di Roma 'Tor Vergata', Via Montpellier 1, I-00133 Roma, Italy. Fax: + 390672596353

E-mail address: santucci@med.uniroma2.it (R. Santucci).
}

heme iron [3-6]. The CL-specific peroxidase action acquired by membrane-bound cyt $c$ in the early stages of apoptosis, which initiates the protein pro-apoptotic activity, is critical for cells; CL peroxidation induces cyt $c$ release into the cytosol and favors the accumulation of products releasing pro-apoptotic factors [7-10]. This explains why considerable effort has been made over recent years to clarify the mechanisms governing the cyt $c-\mathrm{CL}$ interaction and the (cyt $c-\mathrm{CL}$ ) complex stability.

Two models have been proposed to describe the process leading to (cyt $c-\mathrm{CL}$ ) complex formation. Both consider that, upon binding, one acyl chain of CL protrudes in the protein interior. However, one model identifies the hosting region in the hydrophobic channel close to the invariant residue N52 [3], whereas the other in the M80-containing loop [4]. The fact that the CL liposomes bind cyt $c$ at two distinct binding sites of the protein $[5,11]$ led to the proposal that two acyl chains of $\mathrm{CL}$ (instead of one, as suggested by the two above mentioned models) may protrude into cyt $c$ [11]. This event is sterically permitted in view of the unique structure of CL which, contrary to the other phospholipids constituting the mitochondrial membrane, possesses four (instead of two) acyl chains [6]. This hypothesis is supported by the fact that CL is 
the only phospholipid of the mitochondrial membrane which binds the protein firmly [12,13].

In this paper we investigate the interaction of CL liposomes with ferric cyt $c$ obtained from two distinct sources, horse heart and yeast. The two proteins have a similar tertiary architecture [14-17], but yeast cyt $c$ displays a significantly lower stability than the protein from horse, it does not bind ATP and, contrary to horse cyt $c$, lacks pro-apoptotic activity $[18,19]$. The CL-cyt $c$ binding reaction has been investigated in the absence and in the presence of ATP and sodium chloride, two compounds that significantly influence the (horse cyt $c$ )-CL binding process [5]. The data clearly show that the two proteins behave differently. Although the horse cyt $c-C L$ interaction is strongly influenced by the two effectors, no effect is observed for the binding reaction of $\mathrm{CL}$ liposomes with yeast cyt $c$. In particular, the fact that sodium chloride dissociates the (horse cyt $c$ )-CL complex but exerts no influence on the (yeast cyt $c$ )-CL complex, suggests that the complex behavior strictly depends on the type of protein utilized. The different behavior shown by the two cytochromes is explained on the basis of the different local structure of the regions involved in the interactions with either CL or ATP.

\section{Materials and methods}

\subsection{Materials}

Horse heart cyt c (type VI, oxidized form) and cardiolipin, as sodium salt from bovine heart (approx 98\% purity, lyophilized powder), were obtained from Sigma Chemical Co (St. Louis, MO, USA) and used without further purification. All reagents were of analytical grade.

\subsection{Liposome preparation}

Aqueous dispersions of CL liposomes were prepared according to a procedure described previously [20]. Briefly, a film of lipid was prepared on the inside wall of a round bottom flask by evaporation of a chloroform solution containing the proper amounts of lipid. The films obtained were stored in a dessicator overnight under reduced pressure and then $1 \mathrm{~mL}$ of a $20 \mathrm{mM}$ Hepes buffer solution was added in order to obtain a $2.5 \mathrm{mM}$ lipid dispersion. Solutions were vortex-mixed and then freeze-thawed six times from liquid nitrogen to $30^{\circ} \mathrm{C}$. Dispersions were then extruded (10 times) through a $100 \mathrm{~nm}$ polycarbonate membrane. Extrusions were carried out at $30^{\circ} \mathrm{C}$. The vesicle size was determined by light scattering measurements. In the buffer, the vesicle diameter is approximately $130 \mathrm{~nm}$; the diameter slightly increases to $140 \mathrm{~nm}$ in the presence of $0.5 \mathrm{M} \mathrm{NaCl}$.

\subsection{Yeast iso-1-cyt c expression and purification}

The expression plasmid pBTRI harboring both the yeast iso-1-cyt $c$ gene (CYC1) and heme lyase gene (CYC3), was a kind gift from A. Grant Mauk of British Columbia University, Vancouver. The CYC1 gene encodes the Cys102Thr variant of yeast iso-1-cyt $c$. In this variant protein dimerization is precluded and the ferricytochrome autoreduction rate is greatly diminished. The optical and electrochemical properties of the variant are indistinguishable from those of the wt protein [21]. Bacterial expression and purification of the recombinant proteins were conducted essentially as described in [22] with minor modifications.

Escherichia coli strain JM 109 containing the pBTRI plasmid was grown at $37^{\circ} \mathrm{C}$ in $2 \mathrm{~L}$ of $\mathrm{SB}$ medium containing $100 \mu \mathrm{g} / \mathrm{mL}$ ampicillin until an absorbance of 0.3 at $600 \mathrm{~nm}$ was achieved. Induction was accomplished by adding IPTG (isopropyl-ß-D-thiogalactopyranoside) to a final concentration of $0.75 \mathrm{mM}$. Cells were incubated at $37^{\circ} \mathrm{C}$ overnight, harvested by centrifugation and frozen at $-80^{\circ} \mathrm{C}$. After thawing, the reddish pellets were re-suspended in $50 \mathrm{mM}$ Tris $\mathrm{pH} 8.0$ (3-4 mL per gram of wet cells). Lysozyme $(1 \mathrm{mg} / \mathrm{mL})$ and DNase $(5 \mu \mathrm{g} / \mathrm{mL})$ were added to the homogenized cells. The suspension was left in ice for $1 \mathrm{~h}$ and then sonicated for $1 \mathrm{~min}$, at medium intensity and $50 \%$ duty cycle. After centrifugation for $30 \mathrm{~min}$ at $10,000 \mathrm{~g}$ to remove cell debris, the clarified supernatant was dialyzed overnight against $10 \mathrm{mM}$ phosphate buffer $\mathrm{pH} 6.2$ and then loaded on a CM 52 column ( $40 \mathrm{~mL}$ bed volume) equilibrated with the same buffer. Purification was performed by washing the column with one volume of $45 \mathrm{mM}$ phosphate $\mathrm{pH} 6.8$, then one volume $45 \mathrm{mM}$ phosphate $\mathrm{pH}$ $6.8,75 \mathrm{mM} \mathrm{NaCl}$ and by eluting the protein with one volume of $45 \mathrm{mM}$ phosphate $\mathrm{pH} 6.8,250 \mathrm{mM} \mathrm{NaCl}$. After purification, the recombinant $\mathrm{C} 102 \mathrm{~T}$ variant of yeast iso-1-cyt $c$ (indicated herein as ' $w t$ ' protein) was promptly oxidized by adding a few grains of potassium ferricyanide and then subjected to extensive dialysis against $100 \mathrm{mM}$ phosphate buffer $\mathrm{pH}$ 7.0. The recombinant protein $(\sim 500 \mu \mathrm{M})$ was more than $98 \%$ pure (from SDS-PAGE analysis and reverse phase HPLC, data not shown) and was stored at $-80{ }^{\circ} \mathrm{C}$ in $200 \mu \mathrm{L}$ aliquots.

\subsection{Circular dichroism measurements}

Circular dichroism (CD) measurements were carried out using a Jasco J-710 spectropolarimeter (Tokyo, Japan) equipped with a PC as data processor. The molar ellipticity, $[\Theta]\left(\mathrm{deg} \mathrm{cm} \mathrm{dmol}^{-1}\right)$, is expressed on a molar heme basis in the Soret ( $400-450 \mathrm{~nm}$ ) region. In the far-UV region, measurements were carried out in the $215-250 \mathrm{~nm}$ range.

Binding of cyt $c$ to $\mathrm{CL}$ liposomes (critical micelle concentration, cmc: approx. $1 \mu \mathrm{M}$ [5], as determined by the spectroscopic method based on the fluorescence of 1,6-diphenylhexatriene [23]) was analyzed by following the changes induced in the Soret $\mathrm{CD}$ region $(400-450 \mathrm{~nm})$ of the protein by stepwise addition of a few microliters of $2.5 \mathrm{mM}$ CL solution to a $10 \mu \mathrm{M}$ cyt $c$ solution $\left(\mathrm{V}_{\mathrm{i}}=1.0 \mathrm{~mL} ; 0.5 \mathrm{~cm}\right.$ pathlength cell). The buffer was $25 \mathrm{mM}$ Hepes containing $0.1 \mathrm{mM}$ EDTA, pH 7.0. Dichroic spectra were recorded 5 min after mixing. The same procedure was employed to follow the salt-induced (cyt $c-C L$ ) complex dissociation.

\subsection{Electronic absorption measurements}

Electronic absorption spectra were measured with a double-beam Cary 5 spectrophotometer (Varian, Palo Alto, CA, USA) at $25^{\circ} \mathrm{C}$ using a $5-\mathrm{mm}$ NMR tube and a $600 \mathrm{~nm} / \mathrm{min}$ scan rate. Cyt $c$ concentration was determined on the basis of the extinction coefficient $\varepsilon=106 \mathrm{mM}^{-1} \mathrm{~cm}^{-1}$ at $408 \mathrm{~nm}$.

\subsection{Resonance Raman measurements}

Resonance Raman (RR) spectra were recorded using a 5-mm NMR tube and by excitation with the 406.7-nm line of a $\mathrm{Kr}^{+}$laser (Innova 300 C, Coherent, Santa Clara, CA, USA). Backscattered light from a slowly rotating NMR tube was collected and focused into a triple spectrometer (consisting of two Acton Research SpectraPro 2300i and a SpectraPro $2500 \mathrm{i}$ in the final stage with a 3600 grooves per millimeter grating) working in the subtractive mode, equipped with a liquid-nitrogen-cooled CCD detector. It should be noted that the spectral resolution of the RR spectra cited in the figure captions is that calculated theoretically on the basis of the optical properties of the spectrometer. However, for the moderately broad experimental resonance Raman bands observed in the present study (about $10 \mathrm{~cm}^{-1}$ ), the effective spectral resolution will in general be lower. $\mathrm{RR}$ spectra were calibrated with indene and $\mathrm{CCl}_{4}$ as standards to an accuracy of $1 \mathrm{~cm}^{-1}$ for intense isolated bands. The complex between the CL liposomes and protein was prepared by stepwise addition of $1 \mu \mathrm{L}$ aliquots of $\mathrm{CL}(2.5 \mathrm{mM})$ buffered solution to $50 \mu \mathrm{L}$ of a $30 \mu \mathrm{M}$ protein buffered solution until the desired final CL concentration was achieved giving a 7:1 CL/cyt $c$ molar ratio. The aliquots were added at $20 \mathrm{~min}$ intervals and the spectrum recorded $20 \mathrm{~min}$ after the final addition of $\mathrm{CL}$. The samples in the presence of $\mathrm{NaCl}$ were prepared by 
addition of an aliquot of concentrated $\mathrm{NaCl}$ to obtain the final concentration of $0.5 \mathrm{M}$. For UV-vis and RR measurements, a higher amount of $\mathrm{NaCl}$ concentration was used compared to $\mathrm{CD}$ in order to maintain the $\mathrm{NaCl} / \mathrm{cyt} c$ molar ratio.

\section{7. $\mathrm{pH}$ measurements}

A Crison 2001 pHmeter (Alella, Spain) was used for $\mathrm{pH}$ measurements.

\section{Results}

\subsection{Ferric cyt c}

The UV-vis spectra of native horse heart and yeast cyt $c$ (Fig. 1, Panel A, traces a) display the characteristic Soret band at $409 \mathrm{~nm}, \mathrm{Q}_{1}$ band at $530 \mathrm{~nm}$ and a weak charge-transfer (CT) band at $695 \mathrm{~nm}$, indicative of the Met-His axial heme coordination. The UV-vis spectra of the native proteins do not change in the presence of $0.5 \mathrm{M} \mathrm{NaCl}$ (Fig. 1, Panel A, traces b) as also confirmed by the RR spectra. The high frequency region spectra of horse heart and yeast cyt $c$ are identical to those obtained in the absence of $\mathrm{NaCl}$, with the typical frequencies of the RR marker bands of a Met-Fe-His low spin heme [1372 $\left(v_{4}\right), 1502$ $\left(v_{3}\right), 1584\left(v_{2}\right)$, and $1635\left(v_{10}\right) \mathrm{cm}^{-1}$ ] (Fig. 1, Panel B, traces a, b). In the low frequency region the RR spectra of horse heart and yeast cyt $c$ are extremely crowded, as a consequence of the saddle-distortion of the heme group (Fig. 2, traces a). Addition of $\mathrm{NaCl}$ does not alter the RR spectra (Fig. 2, traces a, b) as also confirmed by the absence of any discernible change in the difference spectra obtained between the native protein(s) in the absence and in the presence of $\mathrm{NaCl}$ (Fig. 2, trace b-a). Therefore, the interactions of the heme group with the surrounding polypeptide, which are responsible for the saddledistortion in the native proteins, are not altered by the ionic strength. The two proteins also show similar well-defined far-UV dichroic spectra (not shown) typical of proteins with $\alpha$-helix secondary structure. As shown by the Soret CD spectra ( $400-450 \mathrm{~nm}$ ) illustrated in Fig. 3, the 416-nm dichroic band of yeast iso-1-cyt $c$ is weaker than that of the equine protein. This suggests that the two proteins have similar but not identical heme pocket structures. In hemoproteins, the Soret $C D$ spectrum is strictly correlated with the heme pocket conformation [24]. In particular, for cyt $c$ the 416-nm Cotton effect is associated to the F82- and M80-heme interaction and is considered a probe of the environment on the M80 side of the heme pocket in the native protein $[25,26]$. Changes of the $416-\mathrm{nm}$ dichroic band reflect perturbations of the heme pocket region and, often, the disappearance of the 416-nm signal has been associated with the formation of non-

A

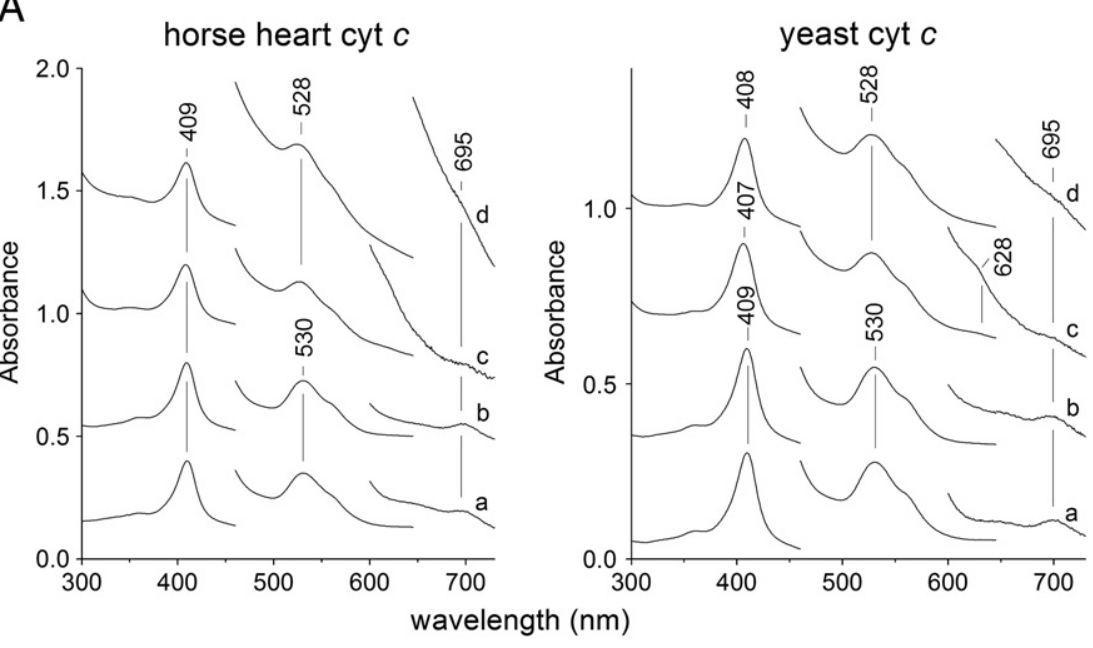

B

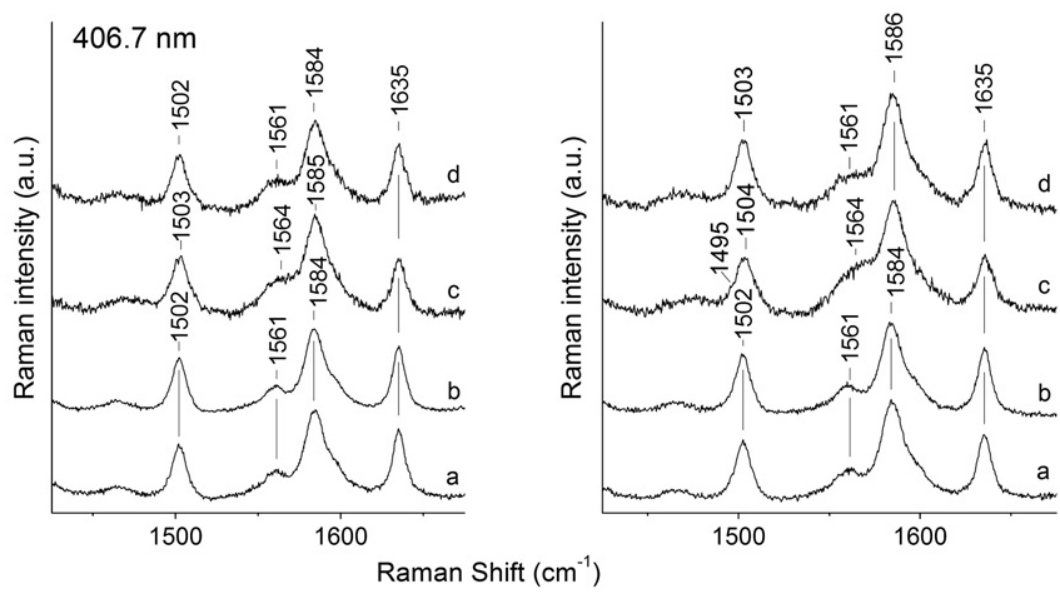

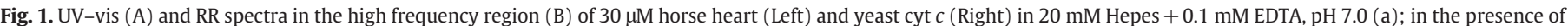

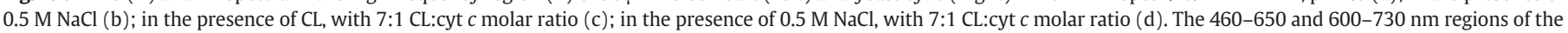

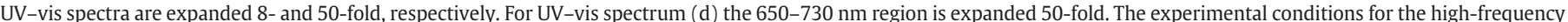

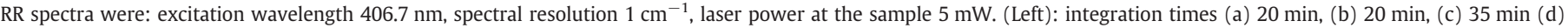

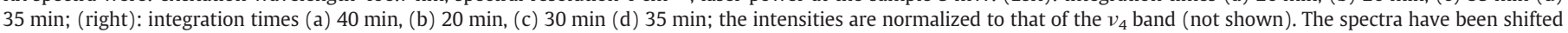
along the ordinate axis to allow better visualization. 


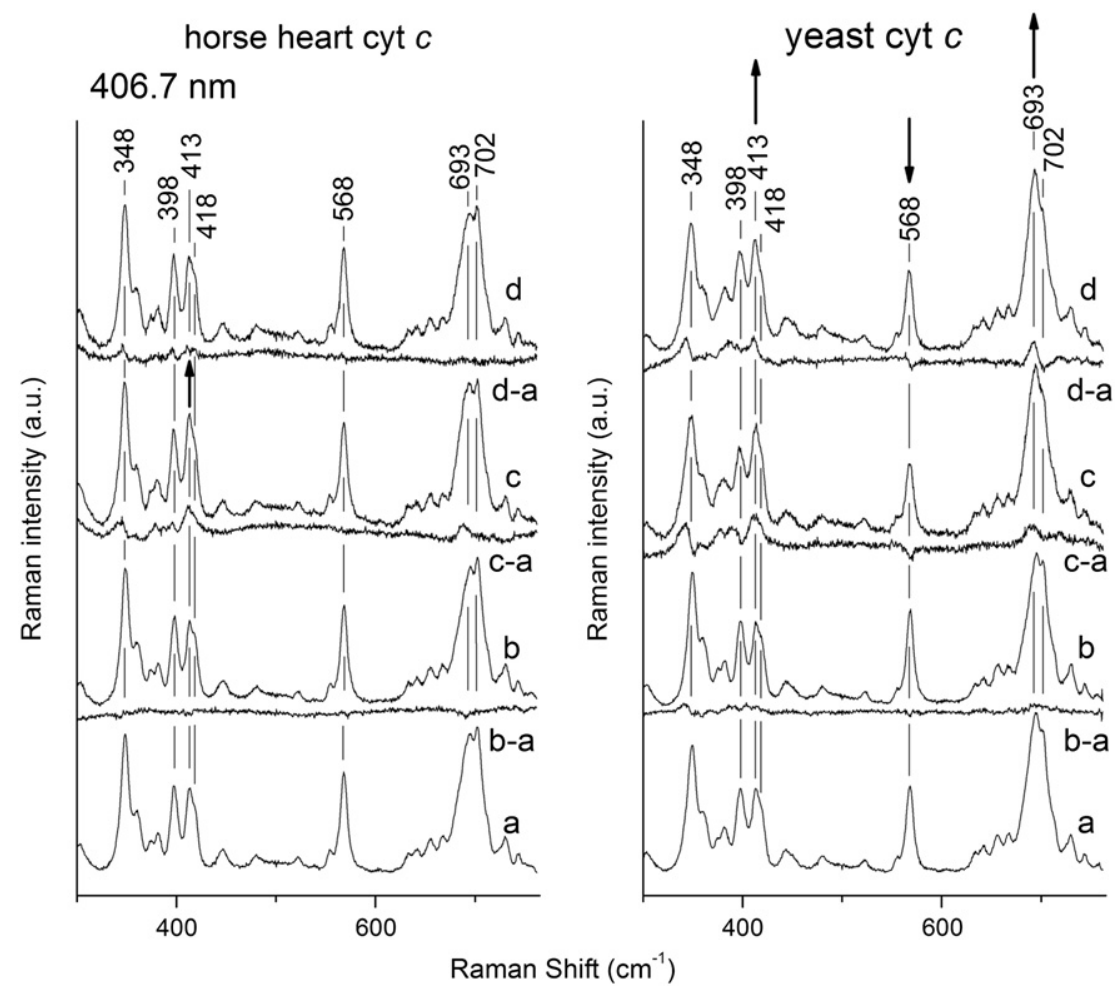

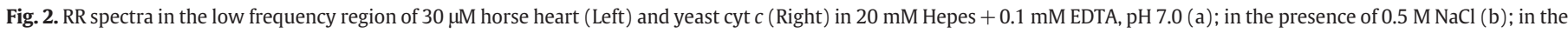

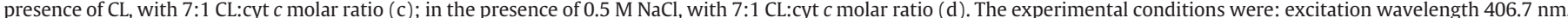

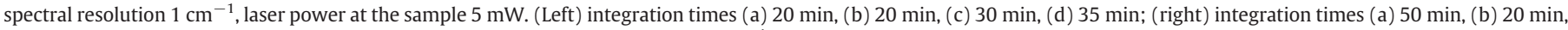

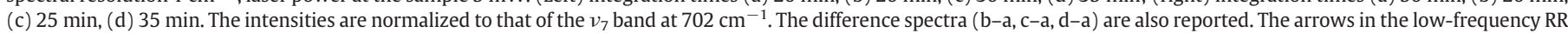

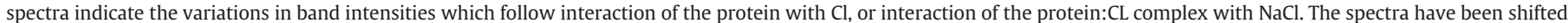
along the ordinate axis to allow better visualization.

native (in some cases unfolded) protein conformations following the rupture of M80-Fe(III) axial bond [26,27].

\subsection{The (cyt c-CL liposome) interaction}

Previous studies on horse ferricyt $c$ have shown that formation of the (CL-cyt c) complex is coupled with tertiary structural changes in the protein and a marked alteration of the heme pocket $[3,5,6,11]$. In this paper, the investigation is extended to yeast iso-1-ferricyt $c$. Fig. 3 shows

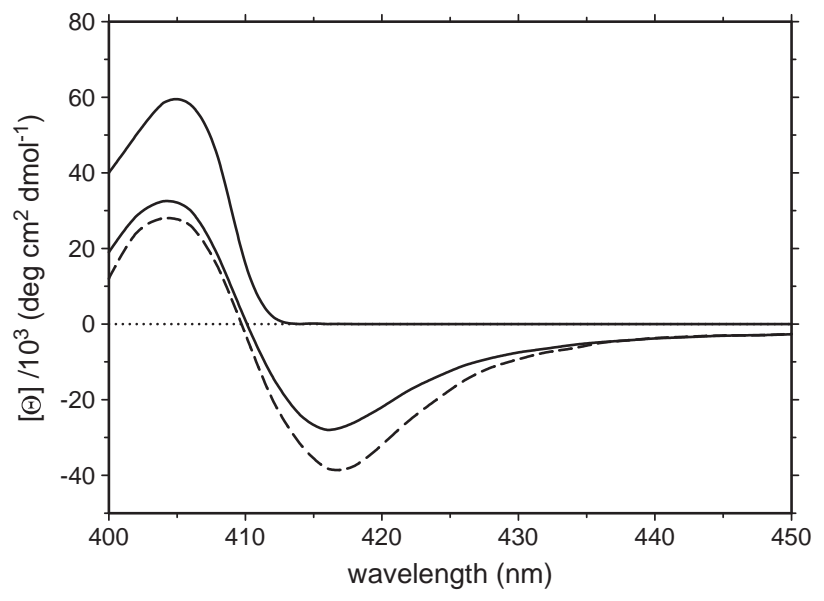

Fig. 3. Soret $C D$ spectrum of horse (-----) and yeast ( middle spectrum) ferricyt c. The dichroic spectrum of the (cyt c-CL) complex obtained after the addition of $60 \mu \mathrm{M}$ CL liposomes (_ upper spectrum) is also shown. Note that CL-bound horse and CL-bound yeast ferricyt $c$ give rise to very similar CD spectra. Protein concentration: $10 \mu \mathrm{M}$. Experimental conditions: $25 \mathrm{mM}$ Hepes + 0.1 mM EDTA, pH 7.0. The temperature was $25^{\circ} \mathrm{C}$. the Soret $\mathrm{CD}$ spectra of the two proteins recorded at neutral $\mathrm{pH}$ in the absence and presence of CL unilamellar liposomes (cmc: $\sim 1 \mu \mathrm{M}$ [3]). The dramatic decrease of the 416-nm dichroic band, observed upon addition of CL liposomes in solution (6:1 CL/cyt $c$ molar ratio), indicates that the protein binds the lipid and that this interaction alters the heme pocket environment. As shown in Fig. 4, the stepwise addition of CL liposomes to either horse or yeast cyt $c$ to form the (cyt $c-\mathrm{CL}$ ) complex gives rise to twotransition curves. In accord with previous reports $[5,6]$, this indicates that cyt $c$ binding to CL occurs via (at least) two distinct sites having different

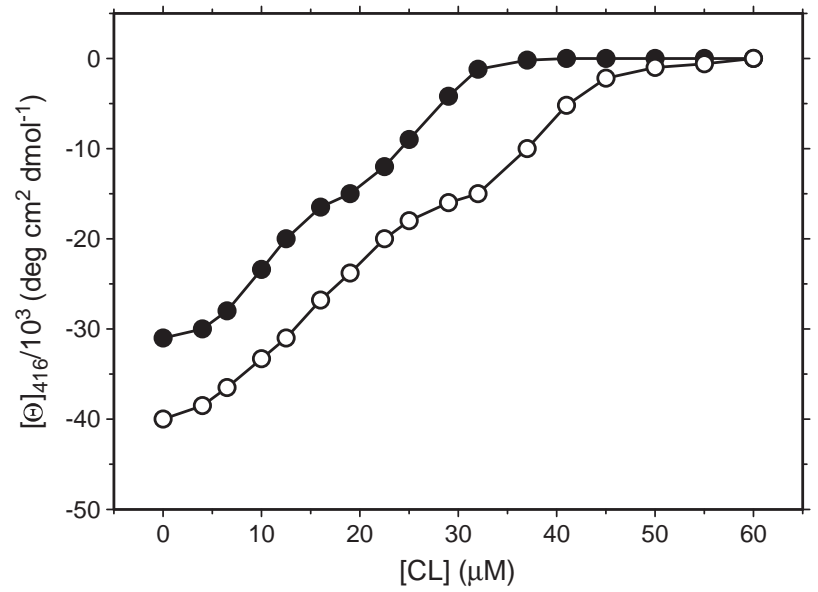

Fig. 4. Titration curves of CL binding to ferricyt $c$. Changes (expressed as \%) of the $416 \mathrm{~nm}-\mathrm{Cotton}$ effect induced by stepwise addition of CL liposomes (cmc: approx. $1 \mu \mathrm{M}$ [3]) to a $10 \mu \mathrm{M}$ horse $(O)$ or yeast $(\bullet)$ cyt $c$ solution. The $416 \mathrm{~nm}$ ellipticity values of the spectra of Fig. 3 were taken as the end points of the binding transition. Experimental points are the average of at least three measurements. Other conditions are as reported for Fig. 3. 
affinity for the phospholipid. In the graph, the position of the titration curves suggests that yeast cyt $c$ binds $\mathrm{CL}$ with higher affinity; the midtransition concentration values $\left(C_{1 / 2}\right)$ are $10 \mu \mathrm{M}$ and $16 \mu \mathrm{M}$ (first transition) and $25 \mu \mathrm{M}$ and $38 \mu \mathrm{M}$ (second transition) for yeast and horse cyt $c$, respectively. The higher affinity of yeast cyt $c$ for $\mathrm{CL}$ may depend on several factors, such as its higher flexibility and/or lower stability with respect to equine cyt $c$ (denaturation free energy, $\Delta \mathrm{Go}_{\mathrm{D}}: 22.5 \mathrm{~kJ} / \mathrm{mol} \mathrm{vs}$ $33.5 \mathrm{~kJ} / \mathrm{mol}$, at $\mathrm{pH} 7.0$ and $25^{\circ} \mathrm{C}$ [28]). CD spectra of horse and yeast cyt $c$ carried out in the near-UV region $(270-300 \mathrm{~nm}$, Fig. S-1) remain unchanged after addition of CL (6:1 CL-cyt $c$ molar ratio) in solution, suggesting that the hydrophobic microenvironment surrounding W59 is nor altered upon (cyt c-CL) complex formation, for both proteins. Further, protein denaturation can be ruled out since no significant changes are observed in the far-UV CD (and, thus, in the $\alpha$-helix content) of CL-bound (yeast and horse) cyt $c$ as compared to the free protein (Fig. S-2).

Upon CL addition a clear increase in scattering occurs in the UV-vis spectra of both (horse cyt $c-\mathrm{CL}$ ) and (yeast cyt $c-\mathrm{CL}$ ) complexes (Fig. 1, Panel A, traces c), which could be at least in part the origin of the spectral variations observed. In both the UV-vis spectra a $2 \mathrm{~nm}$ blue-shift of the $Q_{1}$ band and loss of intensity of the CT band at $695 \mathrm{~nm}$ are observed. However, spectral variations are more significant for yeast cyt $c$, where the interaction with $C L$ also causes a $2 \mathrm{~nm}$ blue-shift of the Soret band and the appearance of a new charge transfer band (CT1) at $628 \mathrm{~nm}$ (Fig. 1, Panel A right, trace c). These spectral changes are consistent with the partial conversion of the protein to nonnative high spin states.

The most significant aspect of the high frequency RR spectra of the (cyt c-CL) complexes (Fig. 1, Panel B, traces c) is the upshift of the most intense bands compared to the frequencies typical of the low spin Met-Fe-His coordination. The upshift is particularly evident for the (yeast cyt $c-C L$ ) complex (Panel B right, trace $c$ ), where a RR band due to a five coordinated high spin is also observed at $1495 \mathrm{~cm}^{-1}$ [6]. Similar changes have been previously observed also upon binding of ferric horse cyt-c to anionic dioleoyl-phosphatidylglycerol (DOPG) vesicles [29]. The pronounced saddle-distortion [30] of the heme group in native cyt $c$ is responsible of the lower core size marker bands frequencies in the RR spectrum compared to planar heme proteins, which display an inverse correlation between RR band frequencies and the porphyrin core size [31-33]. Therefore, the upshift in the RR bands observed upon CL binding is indicative of the conversion to a more planar heme.

The RR low frequency region of the (horse cyt $c-C L$ ) complex (Fig. 2, left, trace c) is marked by a slight increase in the relative intensity of the band at $413 \mathrm{~cm}^{-1}$. More significant changes are observed in the RR spectrum of the (yeast cyt c-CL) complex (Fig. 2, right, trace $\mathrm{c}$ ). The increase in the relative intensity of the band at $413 \mathrm{~cm}^{-1}$ is more pronounced compared to equine cyt $c$, the band at $693 \mathrm{~cm}^{-1}$ is intensified compared to that at $702 \mathrm{~cm}^{-1}$ and the decrease of intensity of the out-of-plane mode $\gamma_{21}$ at $568 \mathrm{~cm}^{-1}$ is observed. These changes, clearly observed as positive and negative peaks, respectively, in the difference spectra (Fig. 2, trace c-a), are very similar to those found upon mutation of cyt $c$ histidines, binding of oleic acid to cyt $c$ or following, the alkaline transition of cyt $c$ [34]. Therefore, in analogy with these cases, the changes observed for the (yeast cyt $c-C L$ ) complex [6] are likely due to a change of axial coordination that results in a less distorted heme $[18,27,28,35,36]$ in which a lysine residue likely replaces M80 at the sixth coordination position of the heme iron. A similar conclusion can be drawn for the (horse cyt $c-\mathrm{CL}$ ) complex.

\subsection{Effect of ionic strength on complex formation/dissociation}

Previous studies on horse ferricyt $c$ have shown that ionic strength affects the protein-CL interaction [5,11], which is strongly decreased in the presence of sodium chloride $(\geq 0.1 \mathrm{M})$. In this paper we compare the protein-CL binding of ferric cyt from horse and yeast.
In accord with a previous study [5], ionic strength modulates the horse cyt $c-\mathrm{CL}$ interaction. As shown in Fig. 5, in the presence of $0.15 \mathrm{M} \mathrm{NaCl}$, only a small fraction (about 10\%) of horse cyt $c$ binds to the phospholipid and the binding curve strongly differs from that obtained at low ionic strength. Conversely, the yeast cyt $c-\mathrm{CL}$ interaction is much less influenced; in this case, the curve shape is similar (although not identical) to that determined at low ionic strength, and the midtransition concentration values $\left(C_{1 / 2}\right)(12 \mu \mathrm{M}$ for the first transition and $32 \mu \mathrm{M}$ for the second transition) indicate that in the reaction with $\mathrm{CL}$ the low affinity binding site is the more sensitive to the ionic strength.

The influence of the ionic strength on the (ferricyt $c-\mathrm{CL}$ ) complex stability was also investigated. The step-by-step addition of salt to the (cyt $c-\mathrm{CL}$ ) complex solution induces gradual dissociation of the (horse cyt $c-C L$ ) complex (Fig. 6). The negative 416-nm dichroic signal, typical of the free protein, is almost fully recovered upon addition of $0.15 \mathrm{M}$ salt concentration. By contrast, no salt effect is observed for the (yeast cyt $c-\mathrm{CL}$ ) complex; the dichroic signal remains unchanged even in the presence of a $0.2 \mathrm{M}$ salt concentration. If the salt concentration is significantly increased, the 416-nm dichroic signal slightly changes; at $0.5 \mathrm{M}$ salt concentration, approx. $28 \%$ of complex is dissociated.

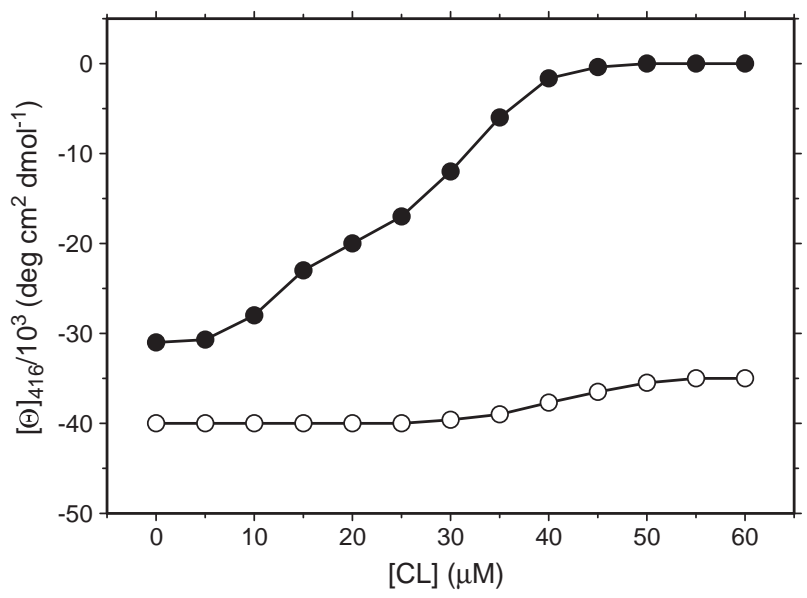

Fig. 5. Effect of ionic strength on the ferricyt $c-C L$ binding reaction. Changes of the $416 \mathrm{~nm}$ dichroic signal induced by addition of CL liposomes to a $10 \mu \mathrm{M}$ horse $(O)$ or yeast $(\bullet)$ cyt $c$ solution in the presence of $0.15 \mathrm{M}$ sodium chloride. Experimental points are the average of at least three measurements. Other conditions are as reported for Fig. 3.

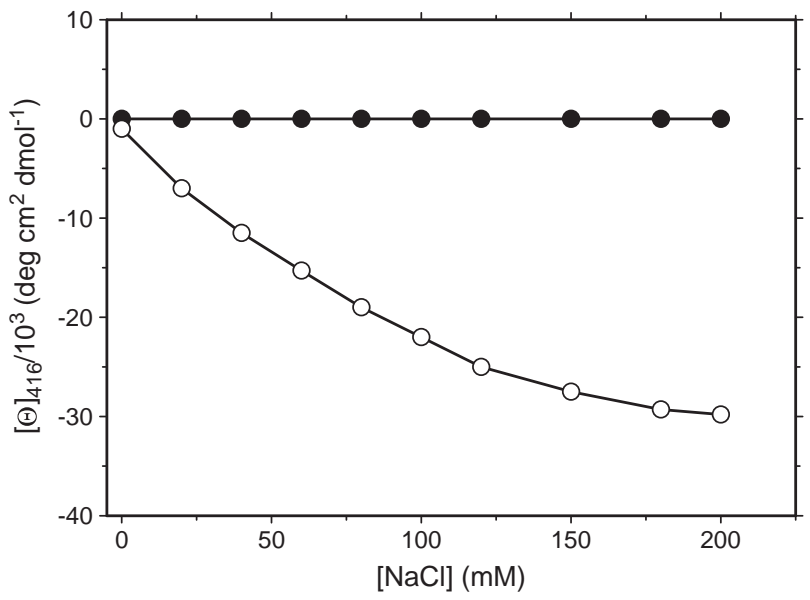

Fig. 6. $\mathrm{NaCl}$-induced dissociation of the ferricyt $c-\mathrm{CL}$ complex. Changes of the $416 \mathrm{~nm}$ Cotton effect induced by stepwise addition of $\mathrm{NaCl}$ to a $10 \mu \mathrm{M}$ horse $(O)$ or yeast $(\bullet)$ cyt $c$ solution containing $60 \mu \mathrm{M}$ CL liposomes. Experimental points are the average of at least three measurements. Other conditions are as reported for Fig. 3. 
Under the same conditions, the (horse cyt $c-\mathrm{CL}$ ) complex dissociation is close to $100 \%$. Such behavior not only reflects structural differences present in horse and yeast cyt $c$ in the proximity of the binding site(s) for $\mathrm{CL}$, but also suggests that the yeast cyt $c-C L$ electrostatic interactions are significantly stronger.

Figs. 1 and 2 compare the UV-vis and RR spectra of the (cyt c-CL) complexes for a $\mathrm{CL}$ :Cytc ratio of 7:1 with and without $0.5 \mathrm{M} \mathrm{NaCl}$ (traces $\mathrm{d}$ and $\mathrm{c}$ ). Addition of $\mathrm{NaCl}$ to the cyt $\mathrm{c}$ - and $\mathrm{CL}$-containing solutions causes a further increase of the scattering that may prevent minor changes in the UV-vis spectrum from being observed (see below). In fact, unlike the (horse cyt c-CL) complex, a 1-nm red shift of the Soret band and a decrease of intensity of the CT1 band are observed upon $\mathrm{NaCl}$ addition in the case of the (yeast cyt $c-\mathrm{CL}$ ) complex. This suggests that, for yeast cyt $c$, there is partial recovery of the low spin coordination. However, addition of $\mathrm{NaCl}$ to the (horse cyt $\mathrm{c}-\mathrm{CL}$ ) complex causes a downshift of the most intense bands of the high frequency region RR spectrum to the frequencies typical of the low spin M80-Fe-H18 (Fig. 1 Panel B left, trace d) and a decrease of the intensity of the band at $413 \mathrm{~cm}^{-1}$ (Fig. 2, left, trace d, and difference spectrum d-a). Therefore, a reversion to the native protein Met-His coordination appears to have occurred. Minor variations are also induced by $\mathrm{NaCl}$ in the $\mathrm{RR}$ spectrum of the (yeast cyt $c-C L$ ) complex, where the disappearance of the $1495 \mathrm{~cm}^{-1}$ band and the downshift of the $\nu_{3}$ (from 1504 to $1503 \mathrm{~cm}^{-1}$ ) and of the $\nu_{11}$ (from 1564 to $1561 \mathrm{~cm}^{-1}$ ) bands are observed (Fig. 1, Panel B, right, trace d). The RR spectrum in the low frequency region does not change in the presence of $\mathrm{NaCl}$. Therefore, the axial coordination of the (yeast cyt $\mathrm{c}-\mathrm{CL}$ ) complex is only slightly affected by the increase in ionic strength. The His-Lys heme coordination population induced upon CL binding to the protein appears unaffected by the presence of $\mathrm{NaCl}$. The presence of $\mathrm{NaCl}$ only affects the amount of the high spin population.

\subsection{Effect of ATP on complex formation/dissociation}

Previous studies have shown that, contrary to yeast, horse cyt $c$ possesses (at least) one binding site with high affinity for ATP [18,19,37]. This is correlated with the critical role played by cyt $c$ in cell apoptosis. As reported earlier, ATP (at $10-15 \mathrm{mM}$ concentration) dissociates the (horse cyt c-CL) complex and induces release of free cyt $c$ in solution [5]. This gives the nucleotide a key role in the apoptotic process, since it facilitates the exit of free cyt $c$ from the mitochondrion at the early stages of the apoptotic process. The titration curves reported in Fig. 7 refer to the binding of CL vesicles to yeast and horse cyt $c$ in the presence of (5-

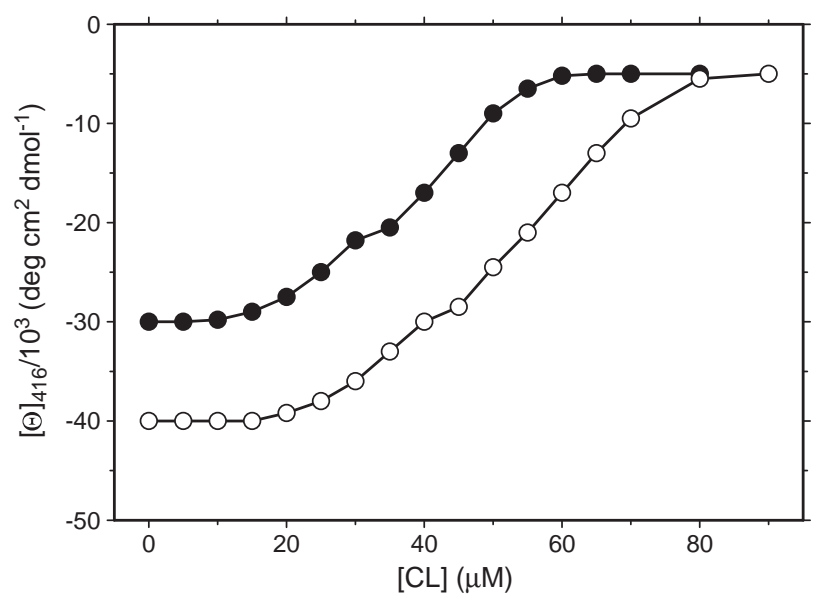

Fig. 7. Effect of ATP on formation of the ferricyt c-CL complex. Changes of the $416 \mathrm{~nm}$ dichroic signal induced by addition of CL liposomes to a $10 \mu \mathrm{M}$ horse $(\mathrm{O})$ or yeast $(\bullet$ cyt $c$ solution, in the presence of $5 \mathrm{mM}$ ATP. Experimental points are the average of at least three measurements. Increase of ATP concentration up to $10 \mathrm{mM}$ after titration produces no effect on the CD signal. Other conditions are as reported for Fig. 3.
$10 \mathrm{mM}$ ) ATP. As in the graphs of Figs. 4 and 5, the experimental points were determined measuring the variation of the 416-nm Cotton effect provoked by the stepwise addition of CL to a cyt $c$ solution. Comparison of Figs. 4 and 7 clearly indicates that both horse and yeast cyt $c$ are influenced by the nucleotide. However, the larger shift of the binding curve towards higher CL concentrations reveals that the influence of ATP is stronger for the (horse cyt $c$ )-CL binding process.

Moreover, for horse cyt $c$, as previously reported [5,19], the addition of (5-10 mM) ATP in solution induces (approx. 40\%) complex dissociation in agreement with Tuominen et al. [38]. In fact, although the latter experiments were carried out under different conditions, (1-3 mM) concentration of ATP significantly altered the tertiary conformation of phosphatidylglycero-bound horse cyt $c$. Therefore, although 1-3 mM ATP is not enough to induce dissociation, it is sufficient to destabilize the complex.

On the whole, the data support the view that there is a competition between ATP and the phospholipid to bind cyt $c$. This conclusion is also supported by the lack of ATP-influence on the stability of yeast cyt $c-\mathrm{CL}$ complex (data not shown), since in yeast cyt $c$ the absence of a specific high affinity site for ATP favors the binding of CL to cyt $c$.

\section{Discussion}

In mitochondrion, a small portion (about 15\%) of cyt $c$ is tightly bound to the membrane while the remainder is free or loosely bound $[1,2]$. The unbound cyt $c$ participates in eT and actively prevents oxidative stress, while membrane-bound cyt $c$ is mainly involved in cell death. To initiate the apoptotic process the protein acquires peroxidase activity, an event which favors protein dissociation from the mitochondrial membrane $[9,39,40]$ and the subsequent release of cyt $c$ into the cytosol. Among the phospholipids constituting the mitochondrial membrane, $\mathrm{CL}$ is known to bind the hemoprotein [41]. Thus, the cyt $c-\mathrm{CL}$ interaction plays an important role in cells since it modulates the protein behavior determining whether cyt $c$ should perform its 'normal' role in the respiratory chain or, conversely, is to be released into the cytosol where it participates in the apoptotic event (see [40] and references therein). Unlike the equine protein, cyt $c$ from yeast does not participate in cell apoptosis $[16,17]$.

In this study we investigated the interaction of horse and yeast cyt $c$ with CL vesicles, here considered a model for the CL-containing mitochondrial membrane, with the aim of identifying the factors distinguishing equine and yeast cyt $c$ in their reaction with CL liposomes.

The present data clearly show that only the horse cyt $c-C L$ interaction is significantly affected by the ionic strength and ATP (both of which also exert considerable influence on the complex stability). Therefore, when the CL-cyt $c$ binding reaction is investigated, it must be considered that $c$-type cyts purified from different sources may display different behavior.

On the basis of the present results a structurally based hypothesis is suggested to explain the different behavior shown by the two cytochromes. In equine cyt $c$ the binding regions for ATP (i.e., the cluster formed by residues E62, K88, R91; see Fig. 8, panel A) and for $\mathrm{CL}$ (the M80-containing loop [4,6,23]) are very close. The observation that ATP hinders CL binding to the protein may find a rationale in the fact that while cyt c-bound ATP stabilizes the 'native' form of the protein, the cyt $c$-bound CL tends to destabilize it. In fact, CL-bound cyt $c$ possesses a (i) nonnative tertiary structure, (ii) altered heme pocket region, and (iii) ruptured $\mathrm{Fe}(\mathrm{III})-\mathrm{M} 80$ axial bond. Thus, ATP and CL appear to act as strong competitors when they react with horse cyt $c$. Also The ATP-induced dissociation of the (cyt $c-\mathrm{CL}$ ) complex can also be explained by the same mechanism.

By contrast, the region that binds ATP in the equine protein is inaccessible to the solvent in yeast cyt $c$, as a consequence of the interaction with the longer N-terminal tail (see panel B of Fig. 8). As a result, the protein shows no affinity for ATP. 
A
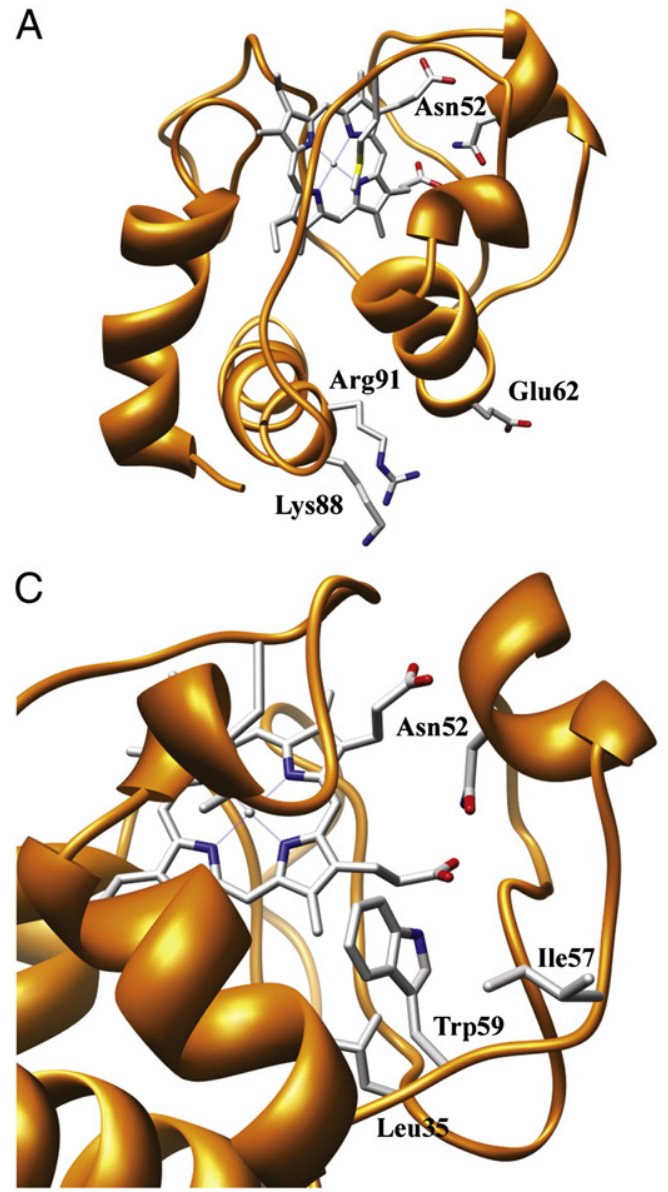

B
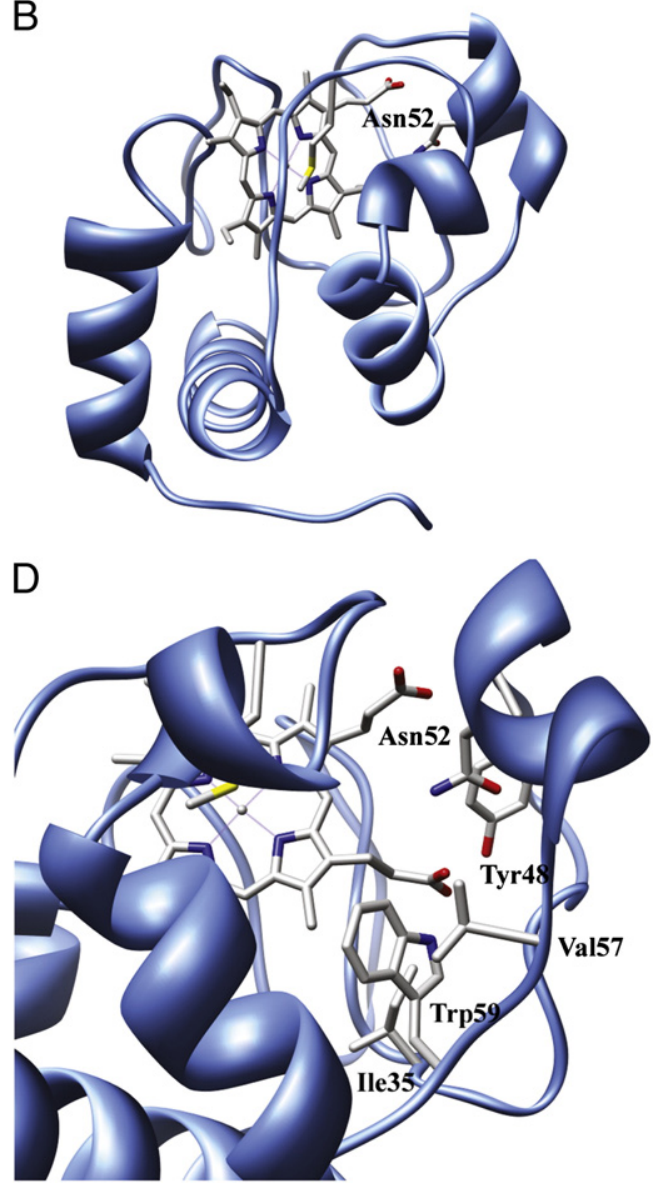

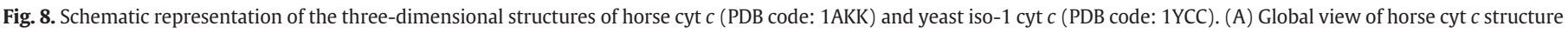

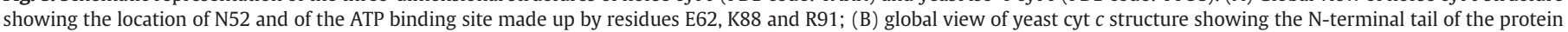

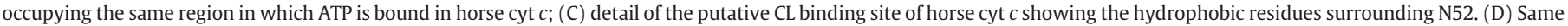
view of panel $C$ for yeast cyt $c$.

The effect exerted by the ionic strength on the ferricyt $c-C L$ interaction is more difficult to explain. Indeed, a comparative analysis of the putative CL binding site in the vicinity of N52 points out structural differences in the two proteins that could, at least in part, explain the different effect exerted by the ionic strength. Compared to the yeast protein, in horse cyt $c$ the region around N52 has a lower hydrophobicity due to a different backbone conformation of the 30-to-60 residues region which causes the displacement of residues 35,48 and 57 (I, Y, and $\mathrm{V}$ residues in yeast cyt $c, \mathrm{~L}, \mathrm{Y}$ and I residues in horse cyt $c$ ) out of the $\mathrm{CL}$ binding site (panels $C$ and D of Fig. 8). The main determinant of this difference appears to be the presence in the horse protein of G56 (in place of N56 in the yeast cyt c; see Figs. 8 and 9), which confers a higher flexibility to the protein backbone. The higher hydrophobicity of the yeast cyt $c$ pocket could favor an increase of protein affinity for CL (as is indeed observed experimentally) and, thus, a decreased propensity of the complex to dissociate even at high ionic strength. Furthermore, yeast cyt $c$ has a larger dipole moment than the horse protein (531 Debye in yeast and 253 Debye in horse cyt $c$, as calculated using the Protein Dipole Moments Server described in [42]). Dipole moment values have been shown to be an important factor in determining the interaction of cyt $c$ on charged surfaces [43], and electrostatic in- teractions of the former protein with the negatively charged phosphate groups of CL might be very strong and less affected by ionic strength. Clearly, further investigation is necessary to clarify this point.

In conclusion, the present study not only confirms that the cyt $c-\mathrm{CL}$ binding reaction is a complex event, it also highlights that specific structural factors strongly affect the behavior of cyt $c$ in its reaction with CL liposomes. As noted above, the conformation of defined regions of the protein, although of relatively limited size, can markedly affect the protein behavior (i.e., affinity) giving rise to differences in its interaction with CL to form the (cyt c-CL) complex, and/or the complex stability in the presence of specific effectors such as ionic strength and ATP.

\section{List of abbreviations}

$\begin{array}{ll}\text { CCD } & \text { Charge-Coupled Device } \\ \text { CD } & \text { circular dichroism } \\ \text { CL } & \text { cardiolipin } \\ \text { cmc } & \text { critical micelle concentration } \\ \text { CT } & \text { charge transfer }\end{array}$

$$
\begin{aligned}
& \text { Yeast 30-PNLHGIFGRHSGQAEGYSYTDANIKKNVLWD-60 } \\
& \text { Horse 30-PNLHGLFGRKTGQAPGFTYTDANKNKGITWK-60 }
\end{aligned}
$$

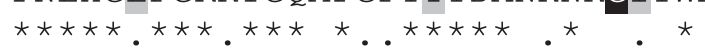

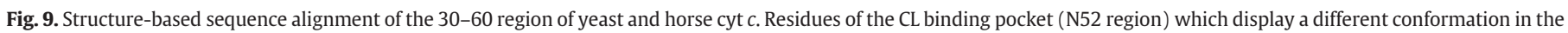
two proteins (see also Fig. 8, panels C and D) are shaded in gray. G56, responsible for the higher flexibility of the horse cyt $c$ backbone in the N52 region, is shaded in black. 
cyt $c \quad$ cytochrome $c$

IPTG isopropyl-ß-D-thiogalactopyranoside

RR resonance Raman

UV-vis Ultraviolet-visible

wt wild type

\section{Acknowledgment}

This work was supported by local Italian grants (ex 60\%) to G.S. and R.S.

\section{Appendix A. Supplementary data}

Supplementary data to this article can be found online at doi:10. 1016/j.jinorgbio.2011.07.022.

\section{References}

[1] M. Schlame, D. Rua, M.L. Greenberg, Prog. Lipid Res. 39 (2000) 257-288.

[2] V.E. Kagan, G.G. Borisenko, Y.Y. Tyurina, V.A. Tyurin, J. Jiang, A.I. Potapovich, V. Kini, A.A. Amoscato, Y. Fujii, Free Radic. Biol. Med. 37 (2004) 1963-1985.

[3] M. Rytömaa, P.K.J. Kinnunen, J. Biol. Chem. 270 (1995) 3197-3202.

[4] E. Kalanxhi, C.J.A. Wallace, Biochem. J. 407 (2007) 179-187.

[5] F. Sinibaldi, L. Fiorucci, A. Patriarca, R. Lauceri, T. Ferri, M. Coletta, R. Santucci, Biochemistry 47 (2008) 6928-6935.

[6] F. Sinibaldi, B.D. Howes, M.C. Piro, F. Polticelli, C. Bombelli, T. Ferri, M. Coletta, G. Smulevich, R. Santucci, J. Biol. Inorg. Chem. 15 (2010) 689-700.

[7] V.E. Kagan, V.A. Tyurin, J. Jiang, Y.Y. Tyurina, V.B. Ritov, A.A. Amoscato, A.N. Osipov, N.A. Belikova, A.A. Kapralov, V. Kini, I.I. Vlasova, Q. Zhao, M. Zou, P. Di, D.A Svistunenko, I.V. Kurnikov, G.G. Borisenko, Nat. Chem. Biol. 1 (2005) 223-232.

[8] Z.T. Schug, E. Gottlieb, Biochim. Biophys. Acta 1788 (2009) 2022-2031.

[9] V.E. Kagan, H.A. Bayir, N.A. Belikova, O. Kapralov, Y.Y. Tyurina, V.A. Tyurin, J. Jiang, D.A. Stoyanovsky, P. Wipf, P.M. Kochanek, J.S. Greenberger, B. Pitt, A.A. Shvedova, G. Borisenko, Free Radic. Biol. Med. 46 (2009) 1439-1453.

[10] P. Caroppi, F. Sinibaldi, L. Fiorucci, R. Santucci, Curr. Med. Chem. 16 (2009) 4058-4065.

[11] M. Rytömaa, K.J. Kinnunen, J. Biol. Chem. 269 (1994) 1770-1774.

[12] M. Rytömaa, P. Mustonen, K.J. Kinnunen, J. Biol. Chem. 267 (1992) 22243-22248

[13] M. Ott, B. Zhivotovsky, S. Orrenius, Cell Death Diff. 14 (2007) 1243-1247.

[14] G.W. Bushnell, G.V. Louie, G.D. Brayer, J. Mol. Biol. 214 (1990) 585-595.

[15] L. Banci, I. Bertini, K.L. Bren, H.B. Gray, P. Sompornpisut, P. Turano, Biochemistry 36 (1997) 8992-9001.

[16] G.V. Louie, G.D. Brayer, J. Mol. Biol. 214 (1990) 527-555.
[17] L. Banci, I. Bertini, H.B. Gray, C. Luchinat, T. Reddig, A. Rosato, P. Turano, Biochemistry 36 (1997) 9867-9877.

[18] F. Sinibaldi, G. Mei, F. Polticelli, M.C. Piro, B.D. Howes, G. Smulevich, R. Santucci, F. Ascoli, L. Fiorucci, Protein Sci. 14 (2005) 1049-1058.

[19] A. Patriarca, T. Eliseo, F. Sinibaldi, M.C. Piro, R. Melis, M. Paci, D.O. Cicero, F. Polticelli, R. Santucci, L. Fiorucci, Biochemistry 48 (2009) 3279-3287.

[20] M.J. Hope, R. Nayar, L.D. Mayer, P.R. Cullis, in: G. Gregoriadis (Ed.), 2nd Ed., Liposome Technology, Vol. 1, CRC Press, Boca Raton, FL, 1992, pp. 123-139.

[21] R.L. Cutler, G.J. Pielak, A.G. Mauk, M. Smith, Protein Eng. 1 (1987) 95-99.

[22] W.B. Pollock, F.I. Rosell, M.B. Twitchett, M.E. Dumont, A.G. Mauk, Biochemistry 37 (1998) 6124-6131.

[23] U. Subuddhi, A.K. Mishra, Colloids Surf. B Biointerfaces 57 (2007) 102-107.

[24] G. Blauer, N. Sreerama, R. Woody, Biochemistry 32 (1993) 6674-6679.

[25] G.J. Pielak, K. Oikawa, A.G. Mauk, M. Smith, C.M. Kay, J. Am. Chem. Soc. 108 (1986) 2724-2727.

[26] R. Santucci, F. Ascoli, J. Inorg. Biochem. 68 (1997) 211-214.

[27] F. Sinibaldi, M.C. Piro, B.D. Howes, G. Smulevich, F. Ascoli, R. Santucci, Biochemistry 42 (2003) 7604-7610.

[28] F. Sinibaldi, B.D. Howes, M.C. Piro, P. Caroppi, G. Mei, F. Ascoli, G. Smulevich, R Santucci, J. Biol. Inorg. Chem. 11 (2006) 52-62.

[29] S. Oellerich, S. Lecomte, M. Paternostre, T. Heimburg, P. Hildebrandt, J. Phys. Chem. B 108 (2004) 3871-3878.

[30] A.M. Berghuis, G.D. Brayer, J. Mol. Biol. 235 (1992) 959-976.

[31] L.D. Spaulding, C.C. Chang, N.-T. Yu, R.H. Felton, J. Am. Chem. Soc. 97 (1975) 2517-2525.

[32] S. Choi, T.G. Spiro, K.C. Langry, K.M. Smith, D.L. Budd, G.N. La Mar, J. Am. Chem. Soc. 104 (1982) 4345-4351.

[33] L.D. Sparks, K.K. Anderson, C.J. Medforth, K. Smith, J.A. Shelnutt, Inorg. Chem. 33 (1994) 2297-2302.

[34] S. Döpner, P. Hildebrandt, F.I. Rosell, A.G. Mauk, J. Am. Chem. Soc. 120 (1998) 11246-11255.

[35] C. Indiani, G. De Sanctis, F. Neri, H. Santos, G. Smulevich, M. Coletta, Biochemistry 39 (2000) 8234-8242.

[36] P. Caroppi, F. Sinibaldi, E. Santoni, B.D. Howes, L. Fiorucci, T. Ferri, F. Ascoli, G. Smulevich, R. Santucci, J. Biol. Inorg. Chem. 9 (2004) 997-1006.

[37] D.B. Craig, C.J.A. Wallace, Protein Sci. 2 (1993) 966-976.

[38] E.K. Tuominen, K. Zhu, C.J. Wallace, I. Clark-Lewis, D.B. Craig, M. Rytomaa, P.K. Kinnunen, J. Biol. Chem. 276 (2001) 19356-19362.

[39] H. Bayir, B. Fadeel, M.J. Palladino, E. Witasp, I.V. Kurnikov, Y.Y. Tyurina, V.A. Tyurin A.A. Amoscato, J. Jiang, P.M. Kochanek, S.T. DeKosky, J.S. Greenberger, A.A. Shvedova, V.E. Kagan, Biochim. Biophys. Acta 1757 (2006) 648-659.

[40] R. Santucci, F. Sinibaldi, A. Patriarca, D. Santucci, L. Fiorucci, Expert Rev Proteomics 7 (2010) 507-517.

[41] R.N. Lewis, R.N. McElhaney, Biochim. Biophys. Acta 1788 (2009) 2069-2079.

[42] C.E. Felder, J. Prilusky, I. Silman, J.L. Sussman, Nucleic Acids Res. 35 (2007) W512-W521.

[43] G. Battistuzzi, M. Borsari, C.A. Bortolotti, G. Di Rocco, A. Ranieri, M. Sola, J. Phys. Chem. B 111 (2007) 10281-10287. 